\title{
Bridging the gap between molecular dynamics simulations and phase-field modelling: dynamics of a $\left[\mathbf{N i}_{x} \mathbf{Z r}_{1-x}\right]_{\text {liquid }}-\mathbf{Z r}_{\text {crystal }}$ Solidification front
}

\author{
Denis Danilov ${ }^{1}$, Britta Nestler ${ }^{1}$, Mohammed Guerdane ${ }^{2}$ and \\ Helmar Teichler ${ }^{2}$ \\ ${ }^{1}$ Institute of Computational Engineering, Karlsruhe University of Applied Sciences, Germany \\ ${ }^{2}$ Institute for Materials Physics, University of Göttingen, Germany
}

Received 29 May 2008, in final form 16 October 2008

Published 15 December 2008

Online at stacks.iop.org/JPhysD/42/015310

\begin{abstract}
Results are presented from phase-field modelling and molecular dynamics simulations concerning the relaxation dynamics in a finite-temperature two-phase crystal-liquid sample subjected to an abrupt temperature drop. Relaxation takes place by propagation of the solidification front under formation of a spatially varying concentration profile in the melt.

The molecular dynamics simulations are carried out with an interatomic model appropriate for the NiZr alloy system and provide the thermophysical data required for setting up the phase-field simulations.

Regarding the concentration profile and velocity of the solidification front, best agreement between the phase-field model and molecular dynamics simulation is obtained when increasing the apparent diffusion coefficients in the phase-field treatment by a factor of four against their molecular dynamics estimates.
\end{abstract}

\section{Introduction}

It is a particular challenge of present materials sciences to provide a scale-bridging link between atomistic descriptions of matter, such as $a b$ initio calculations or molecular dynamics simulations, and meso- and macro-scale approaches such as phase-field modelling or elasticity theory. The different approaches have their individual length scales of optimal applicability, which immediately raises the question for transmitting results from one range to the next. A bridging of the gap between approaches on neighbouring scales can be attempted by exchanging parameters. However, this leaves open the question of equivalence of the treatments, especially in the borderline region of the approaches. Testing the equivalence needs detailed comparison of the predictions of the considered approaches for an appropriate situation. Such a test is of particular importance, as it will prove the range of applicability of the approaches, and it will make obvious whether the considered methods describe well the same aspects of physics, regardless of their inevitably different assumptions and approximations. To carry out such a test, we here compare results from molecular dynamics simulations and phase-field predictions about the propagation of a planar $\left[\mathrm{Ni}_{x} \mathrm{Zr}_{1-x}\right]_{\text {liquid }}-\mathrm{Zr}_{\text {crystal }}$ solidification front. In detail, we consider a situation where an equilibrated twophase crystal-liquid sample with temperature $T$ is driven into a non-equilibrium situation by subjecting it to an abrupt temperature drop $\Delta T$. The subsequent propagation dynamics of the $\left[\mathrm{Ni}_{x} \mathrm{Zr}_{1-x}\right]_{\text {liquid }}-\mathrm{Zr}_{\text {crystal }}$ interface is considered and the changes of the concentration profiles are investigated taking place while the liquid relaxes into the new, lower-temperature equilibrium. Phase-field modelling (e.g. [1]) and molecular dynamics simulations are suited to deal with the relaxation dynamics. There are, however, the questions whether one can apply phase-field modelling down to the range of the atomistic structure and whether isothermal non-equilibrium 
molecular dynamics simulations are capable of properly treating the relaxation dynamics driven by thermodynamic forces. $\mathrm{Ni}_{x} \mathrm{Zr}_{1-x}$ is chosen, as there exists for this system an atomistic model, whose thermodynamic properties are known sufficiently well from a recent molecular dynamics study [2]. Originally the model was designed to analyse glass forming properties of undercooled melts (e.g. [3]). As shown in [2], the Zr-rich part of the $(x, T)$-phase diagram simulated with this model is in good agreement with the experimental diagram [4], in particular regarding the eutectic $\mathrm{Zr}$ concentration of $73 \%$ and $63 \%$ (experimentally $76 \%$ and $64 \%$ ) and the ratio of melting to eutectic temperatures. Moreover, the $\left[\mathrm{Ni}_{x} \mathrm{Zr}_{1-x}\right]_{\text {liquid }}-$ $\mathrm{Zr}_{\text {crystal }}$ liquidus line is known from the molecular dynamics simulations, which is one of the important pre-requisites needed for phase-field modelling of the interface dynamics.

The phase-field approach $[1,5]$ is established as a powerful tool for modelling microstructure formation (dendritic patterns, cellular growth, etc) on meso- and microscopic scales. The phenomenological evolution equations can be derived in a thermodynamically consistent way by demanding that the entropy increases locally for a system, where the internal energy and concentrations are conserved (see for details $[6,7]$ ). The evolution equations contain parameters which describe properties of the interface between different phases. These parameters cannot be derived within the framework of the phenomenological model, and they have to be determined by a separate consideration of the processes at the interface, i.e. by direct experimental measurements or by simulations from other models. At the same time, atomistic (preferably molecular dynamics) computer simulations have become an important tool in providing detailed information about crystallization and melting processes and about the properties of the crystal-melt interface. The atomistic simulations are especially important for the study of a moving crystal-melt interface, because it is in general excessively elaborate to access directly the moving interface in experiments.

In paper [8], a multiscale modelling approach has been suggested, where the interfacial properties are determined by results of molecular dynamics simulations on the atomistic scale [9]. The computed material properties of the solid-liquid interface in pure $\mathrm{Ni}$ are used as input data for the phenomenological phase-field model of dendritic microstructure formation at a mesoscopic scale level. In the case of dendritic growth, the shape and the velocity of a dendrite are very sensitive quantities depending strongly on the interfacial energy and on the kinetic coefficient. The atomistic simulations help to provide more realistic values of those key parameters which have so far been remained too difficult to measure experimentally.

A recent research direction is the development of phasefield crystal models which allow predictions of thermophysical quantities such as surface energies and anisotropies. In [10] the phase-field crystal modelling technique is applied to a bcc-liquid interface and compared with molecular dynamics simulations.

In section 2, this paper continues with a brief description of the molecular dynamics and phase-field method considered for the scale- and model-bridging comparison of the diffusion process and dynamics of the NiZr solidification front. In section 3 , we outline which thermophysical parameters are needed as input data for the phase-field simulations and explain the procedure how they are determined from the atomistic molecular dynamics simulations. Based on the transfer of thermophysical data, the dynamics of the solid-liquid interface simulated with both methods is compared in section 4 and results are finally discussed.

\section{Modelling methods}

\subsection{Molecular dynamics simulation and sample preparation}

The molecular dynamics calculations are carried out as isothermal-isobaric $(N, T, p)$ simulations for a two-phase $\mathrm{Ni}_{x} \mathrm{Zr}_{1-x}$ layer structure. The equations of motion of $N$ particles at temperature $T$ and (zero) external pressure $p$ are numerically integrated by a fifth-order predictor-corrector algorithm with time step $\Delta t_{0}=2.5 \times 10^{-15} \mathrm{~s}$. The temperature is measured in terms of suitable time averages of the kinetic energy. Changes of the temperature are modelled by changing the mean kinetic energy, where in our highly complex system of interacting particles, a continuous exchange between kinetic and potential energy takes place. In the calculations, samples with up to $N=103693$ atoms are considered in an orthorhombic box with cyclic boundary conditions in the three direction of space, with a fluctuating box extension evaluated via Anderson's procedure from a zero-pressure condition.

As in previous studies (cf e.g. [2,3]), we model the interatomic couplings by short-ranged pair potentials $\phi(I J)$ $(I, J \in \mathrm{Ni}, \mathrm{Zr}$ ), aimed at taking care of the electronic $\mathrm{d}$-state interactions and residual local couplings between s- and p-states, and a volume-dependent part $E^{\mathrm{Vol}}$ that provides the electron gas pressure of $\mathrm{s}$ - and p-electrons. For the pair potentials, a short-ranged expression of Stillinger-Weber form [11] is used, adapted to the interactions deduced by Hausleitner and Hafner [12] within their hybridized nearly-free-electrontight-binding-bond model for amorphous binary transition metal alloys. For further details, see, e.g. [2,3].

The interaction model describes fairly well the steric conditions in crystalline phases, as demonstrated for instance by the nearest neighbour distances of the pure $\mathrm{Zr}$ and by the radial distribution functions in undercooled melts. In the case of the intermetallic $\mathrm{Ni}_{50} \mathrm{Zr}_{50}$ compound, the computer model yields a significantly lower energy for the experimentally stable, orthorhombic $B 33$-structure than for the simple $B 1$ or $B 2$ structures, where the calculated $B 33$ cohesion energy of $6.05 \mathrm{eV}$ atom $^{-1}$ [13] compares sufficiently well with its experimental value $5.88 \mathrm{eV}$ atom $^{-1}$. The simulated melting temperature for the $B 33$ structure, $T_{\mathrm{m}}\left(\mathrm{Ni}_{50} \mathrm{Zr}_{50}\right)=1950 \mathrm{~K}$ [13] has to be reduced by about $22 \%$ to get an agreement with the experimental value of $1533 \mathrm{~K}$. Furthermore, the present model yields by MD simulation diffusion coefficients for $\mathrm{Ni}$ in liquid $\mathrm{Ni}_{30} \mathrm{Zr}_{70}\left(D=1.6 \times 10^{-9} \mathrm{~m}^{2} \mathrm{~s}^{-1}\right.$ at $\left.T=1700 \mathrm{~K}\right)$ in good agreement with experimental results for liquid $\mathrm{Ni}_{36} \mathrm{Zr}_{64}$ ( $D \sim 2 \times 10^{-9} \mathrm{~m}^{2} \mathrm{~s}^{-1}$ at $T=1700 \mathrm{~K}$ [14]). Concerning the glassy state, a fair agreement is also obtained between MD simulation results and experimental ones for the system 


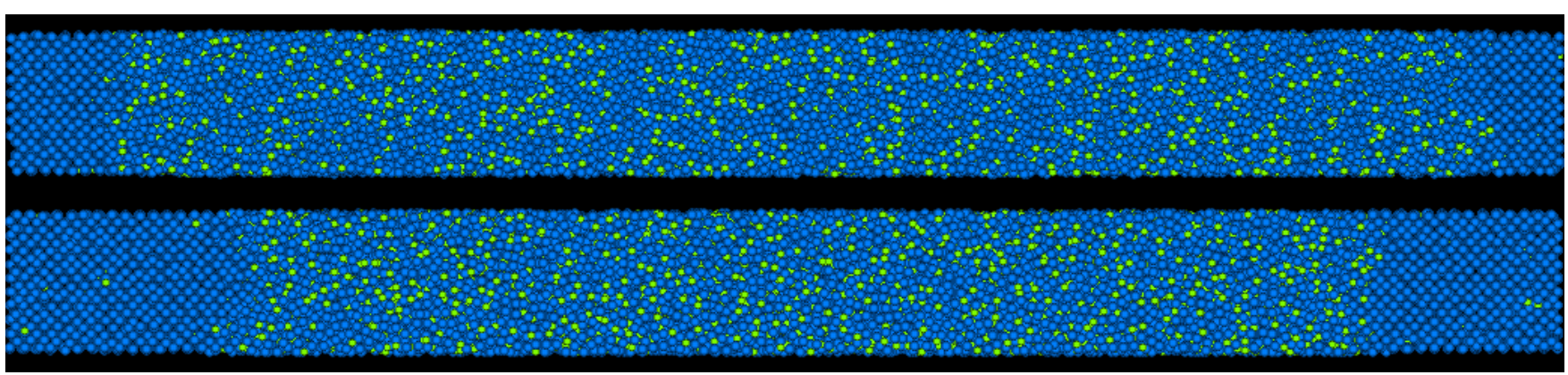

Figure 1. Two images of the crystal-melt system $\mathrm{Ni}_{x} \mathrm{Zr}_{1-x}$. The first structure (upper panel) corresponds to the equilibrium condition at $T=1900 \mathrm{~K}$. The second snapshot shows the interface after $20 \mathrm{~ns}$ crystallization and a cooling of the sample down to $T=1700 \mathrm{~K}$.

(This figure is in colour only in the electronic version)

$\mathrm{Ni}_{50} \mathrm{Zr}_{50}$ (simulation: $D_{0}=5 \times 10^{-7} \mathrm{~m}^{2} \mathrm{~s}^{-1}, Q=1.2 \mathrm{eV}$ [15, 16]); experiment: $D_{0}=1.7 \times 10^{-7} \mathrm{~m}^{2} \mathrm{~s}^{-1}, Q=$ $1.3 \mathrm{eV}$ [17]). Thus, the interatomic potentials of the MD model give a realistic description of the dynamics in Zr-rich $\mathrm{NiZr}$ alloys, especially for the temperature range (liquid phase) considered in this work. The deviation in the glassy state may be related, to a certain part, to the different degrees of relaxation achieved in MD simulations and experiments.

In our molecular dynamics simulation, the two-phase crystal-liquid structure is prepared as follows: first, a bcc $\mathrm{Zr}$ crystal is equilibrated at $T=1900 \mathrm{~K}$ (melting temperature of the simulation model $T_{\mathrm{m}}=2735 \mathrm{~K}$, experimentally $T_{\mathrm{m}}=$ $2128 \mathrm{~K}$ ). Then, a liquid box is constructed with cross-sectional area in the $y z$ plane equal to that of the equilibrated $\mathrm{Zr}$ crystal box $(10 \times 10$ unit cell lengths). Notation $x, y, z$ mean here and in the following the Cartesian directions, with $x$ orthogonal to the crystal-melt interface. The fraction of $\mathrm{Ni}$ atoms shall be denoted by concentration $c$. This liquid probe is first equilibrated at $T=3000 \mathrm{~K}$ for about $4 \times 10^{5}$ steps $(1 \mathrm{~ns})$, then cooled at a rate of about $10^{12} \mathrm{~K} \mathrm{~s}^{-1}$ down to $T=1900 \mathrm{~K}$ and relaxed again for about $4 \mathrm{~ns}$. Thereby the length of the liquid cells in the $y$ and $z$ directions are held constant at the initial values, while the box length along the $x$ direction changes to obtain the equilibrium condition of zero pressure. The $y z$ surface of the bcc $\mathrm{Zr}$ crystal is then brought into contact with the $y z$ section of the liquid $\mathrm{Ni}_{x} \mathrm{Zr}_{1-x}$. Due to the periodic boundary conditions, two solid-liquid interfaces perpendicular to the $x$-direction are created in the system. The constructed two-phase system is equilibrated until the specific equilibrium Ni concentration of the melt $c_{\mathrm{L}}(T)=0.2175$ is reached, which means equilibrium between crystalline $\mathrm{Zr}$ and liquid $\mathrm{Ni}_{x} \mathrm{Zr}_{1-x}$ as given by the $\left[\mathrm{Ni}_{x} \mathrm{Zr}_{1-x}\right]_{\text {liquid }}-\mathrm{Zr}_{\text {crystal }}$ liquidus line. A change of the composition of the melt occurs, when the crystalline part either grows or shrinks. In order to reduce the equilibration time, the starting composition of the liquid part is chosen close to the equilibrium value.

Figure 1 (upper panel) displays the initial two-phase system equilibrated at $T=1900 \mathrm{~K}$. The temperature dependence of $c_{\mathrm{L}}(T)$ is accessible to simulations by annealing the layered structure at different temperatures. As an example, figure 1 (lower panel) shows the same sample after cooling it down to $T=1700 \mathrm{~K}$ and relaxing it for $20 \mathrm{~ns}$. Clearly visible is an expansion of the crystalline fraction at the reduced temperature on account of the molten part, which implies an increase in $c_{\mathrm{L}}(T)$ under cooling. The results of such equilibration molecular dynamics simulations in the range of temperatures between $T=2100 \mathrm{~K}$ and $T=1500 \mathrm{~K}$ are presented in figure 3. In the simulations, the total length of the sample is $L_{x}=1786.76 \AA$, where the crystal part extends over about $100 \mathrm{bcc}$ unit cells, which corresponds to $400 \AA$. The $c_{\mathrm{L}}(T)$ values fit well to the data of [2], where the range from 1800 to $1250 \mathrm{~K}$ is considered.

\subsection{Phase-field model}

We use the phase-field formulation for alloy solidification that has recently been proposed in [7] for a general class of multicomponent and multiphase systems. The model is based on an entropy functional and the evolution equations are derived in consistency with the first (positive local entropy production) and second (conservation equations) law of thermodynamics. The general case of multiple components is reduced to a binary alloy with components A (solvent) and $\mathrm{B}$ (solute) and to a system of two phases (solid and liquid).

A constant temperature $T$ is considered as a parameter, and the free energy density $f$ is postulated in the form

$$
\begin{aligned}
& f(c, T)=\frac{R T}{v_{\mathrm{m}}}\left(c_{\mathrm{A}} \ln c_{\mathrm{A}}+c_{\mathrm{B}} \ln c_{\mathrm{B}}\right)+\frac{R T}{v_{\mathrm{m}}}\left(c_{\mathrm{A}} \ln F_{\mathrm{A}}(T)\right. \\
& \left.-c_{\mathrm{B}} \ln k_{\mathrm{e}}\right) h(\varphi),
\end{aligned}
$$

where $c_{\mathrm{A}}$ is the concentration in molar fractions of component $\mathrm{A}(\mathrm{Zr}), c_{\mathrm{B}}$ is the concentration in molar fractions of component $\mathrm{B}(\mathrm{Ni}), k_{\mathrm{e}}$ is the equilibrium partition coefficient, $v_{\mathrm{m}}$ is the molar volume and $R$ is the gas constant. The phase-field variable $\varphi$ describes the thermodynamic state of a local volume. The value $\varphi=1$ corresponds to the solid phase, $\varphi=0$ corresponds to the liquid and the function $h(\varphi)=\varphi^{2}(3-2 \varphi)$ is monotonic in the interval $[0,1]$ satisfying the conditions $h(0)=0$ and $h(1)=1$ in the bulk phases. The form of the function $F_{\mathrm{A}}(T)$ is

$$
F_{\mathrm{A}}(T)=\frac{1-C_{\mathrm{L}}(T)}{1-k_{\mathrm{e}} C_{\mathrm{L}}(T)},
$$

and $C_{\mathrm{L}}(T)$ defines the liquidus line of the equilibrium phase diagram.

Considering a planar solid-liquid interface, we write the evolution equations of the phase-field model for isotropic 
kinetics and isotropic surface energies of the solid-liquid interface. The evolution of the phase-field variable $\varphi$ is determined by the partial differential equation

$$
\frac{2 \varepsilon \gamma}{v} \frac{\partial \varphi}{\partial t}=2 \varepsilon \gamma \frac{\partial^{2} \varphi}{\partial x^{2}}-\frac{9 \gamma}{\varepsilon} \frac{\partial g(\varphi)}{\partial \varphi}-\frac{1}{T} \frac{\partial f}{\partial \varphi},
$$

where $g(\varphi)=\varphi^{2}(1-\varphi)^{2}$ is a double well potential, $\gamma$ is the entropy density of the solid-liquid interface, $v$ is the interface mobility and the parameter $\varepsilon$ determines the thickness of the interfacial zone. The concurrence between the first and the second term on the right-hand side of equation (3) generates the diffuse transition zone between the crystal and melt phases. The last term drives the growth.

The diffusion mass transport of the alloy component B is determined by a nonlinear diffusion equation

$\frac{\partial c_{\mathrm{B}}}{\partial t}=\frac{\partial}{\partial x}\left(D(\varphi) \frac{\partial c_{\mathrm{B}}}{\partial x}\right)-\Theta \frac{\partial}{\partial x}\left(D(\varphi) c_{\mathrm{B}}\left(1-c_{\mathrm{B}}\right) \frac{\partial h}{\partial x}\right)$,

where $D(\varphi)=D_{\mathrm{S}} \varphi+D_{\mathrm{L}}(1-\varphi)$ with constant diffusion coefficients $D_{\mathrm{S}}$ and $D_{\mathrm{L}}$ of component $\mathrm{B}$ in the solid and in the liquid phase, respectively. To derive equation (4), the constraint condition $c_{A}+c_{\mathrm{B}}=1$ has been applied. The quantity $\Theta$ denotes the driving force for the redistribution of the alloy components at the solid-liquid interface

$$
\Theta=\ln F_{\mathrm{A}}(T)+\ln \left(k_{\mathrm{e}}\right) .
$$

To solve the evolution equations for the phase-field variable in equation (3) and for the concentration field in equation (4) numerically, we use a finite difference discretization method with a semi-implicit time marching algorithm. The simulation domain is set up with the identical physical expansion as in the molecular dynamics simulations. The numerical grid is accommodated appropriately, so that the solid-liquid interface is resolved on an atomic length scale comparable to the molecular dynamics configurations.

\subsection{Analytical sharp interface model}

In addition to the numerical simulations, we consider an analytical solution to the diffusion limited growth of the solid phase. A two-sided sharp interface formulation allows to provide an estimated description of the dynamics of the interface motion. To derive the model equations, we consider two semi-infinite domains corresponding to the solid and liquid.

Even for the large undercooling of $200 \mathrm{~K}$, effects of solute trapping do not occur in the $\mathrm{Ni}-\mathrm{Zr}$ alloy system at the considered composition. This is due to the fact that the solidification interval, i.e. the difference between the solidus and liquidus curve, extends over applied undercooling for the considered composition (because of extremely small value of the partition coefficient). As a result, a diffusion regime with interface velocity asymptotically decreasing to zero is realized and no steady-state condition can be established.

The domains are connected by a sharp solid-liquid interface located at the coordinate point $x=x_{\mathrm{f}}(t)$ depending on the time variable due to the motion of the interface.
The diffusion fields $c_{\mathrm{S}}$ and $c_{\mathrm{L}}$ of the impurity component in the solid and in the liquid are described by the diffusion equations

$\frac{\partial c_{\mathrm{S}}}{\partial t}=D_{\mathrm{S}} \frac{\partial^{2} c_{\mathrm{S}}}{\partial x^{2}}, \quad x<x_{\mathrm{f}} \quad$ and

$\frac{\partial c_{\mathrm{L}}}{\partial t}=D_{\mathrm{L}} \frac{\partial^{2} c_{\mathrm{L}}}{\partial x^{2}}, \quad x>x_{\mathrm{f}}$

The following conditions are fulfilled in the regions $x \rightarrow \pm \infty$ and at the sharp interface $x_{\mathrm{f}}$ :

$\left.c_{\mathrm{S}}(x, t)\right|_{x \rightarrow-\infty}=c_{\mathrm{S}}^{0} \quad$ and $\left.\quad c_{\mathrm{L}}(x, t)\right|_{x \rightarrow+\infty}=c_{\mathrm{L}}^{0}$,

$-\left.D_{\mathrm{L}} \frac{\partial c_{\mathrm{L}}}{\partial x}\right|_{x=x_{\mathrm{f}}}+\left.D_{\mathrm{S}} \frac{\partial c_{\mathrm{S}}}{\partial x}\right|_{x=x_{\mathrm{f}}}=\left(c_{\mathrm{L}}^{\mathrm{i}}-c_{\mathrm{S}}^{\mathrm{i}}\right) \frac{\mathrm{d} x_{\mathrm{f}}}{\mathrm{d} t}$,

where $c_{\mathrm{L}}^{\mathrm{i}}$ and $c_{\mathrm{S}}^{\mathrm{i}}$ are the equilibrium liquidus and solidus concentration, respectively. Together with the initial conditions $\left.x_{\mathrm{f}}\right|_{t=0}=0,\left.c_{\mathrm{L}}(x, t)\right|_{t=0}=c_{\mathrm{L}}^{0}$ and $\left.c_{\mathrm{S}}(x, t)\right|_{t=0}=c_{\mathrm{S}}^{0}$, equations (6)-(8) describe the diffusion limited growth in the sharp interface approximation.

Assuming that the motion of the interface obeys the selfsimilarity form

$$
x_{\mathrm{f}}=2 \Omega \sqrt{t},
$$

we get from equation (6) the concentration profiles in the solid

$c_{\mathrm{S}}=c_{\mathrm{S}}^{0}+\left(c_{\mathrm{S}}^{\mathrm{i}}-c_{\mathrm{S}}^{0}\right) \frac{\operatorname{erfc}\left(\eta_{\mathrm{S}}\right)}{\operatorname{erfc}\left(\Omega / \sqrt{D_{\mathrm{S}}}\right)}$ with $\eta_{\mathrm{S}}=\frac{x}{2 \sqrt{D_{\mathrm{S}} t}}$

and in the liquid

$c_{\mathrm{L}}=c_{\mathrm{L}}^{0}+\left(c_{\mathrm{L}}^{\mathrm{i}}-c_{\mathrm{L}}^{0}\right) \frac{\operatorname{erfc}\left(\eta_{\mathrm{L}}\right)}{\operatorname{erfc}\left(\Omega / \sqrt{D_{\mathrm{L}}}\right)} \quad$ with $\eta_{\mathrm{L}}=\frac{x}{2 \sqrt{D_{\mathrm{L}} t}}$

From the interface conditions (7)-(8), we obtain an equation for the parameter $\Omega$

$$
\begin{aligned}
& \frac{\left(c_{\mathrm{L}}^{\mathrm{i}}-c_{\mathrm{L}}^{0}\right) \sqrt{D_{\mathrm{L}}}}{\operatorname{erfc}\left(\Omega / \sqrt{D_{\mathrm{L}}}\right)} \exp \left(-\frac{\Omega^{2}}{D_{\mathrm{L}}}\right)-\frac{\left(c_{\mathrm{S}}^{\mathrm{i}}-c_{\mathrm{S}}^{0}\right) \sqrt{D_{\mathrm{S}}}}{\operatorname{erfc}\left(\Omega / \sqrt{D_{\mathrm{S}}}\right)} \exp \left(-\frac{\Omega^{2}}{D_{\mathrm{S}}}\right) \\
& \quad=\sqrt{\pi}\left(c_{\mathrm{L}}^{\mathrm{i}}-c_{\mathrm{S}}^{\mathrm{i}}\right) \Omega .
\end{aligned}
$$

By introducing the variable $\Lambda$ and the relation $\Omega=$ $\Lambda \sqrt{D_{\mathrm{L}}}$, we can rewrite equation (12) to

$$
\begin{aligned}
& \frac{\left(c_{\mathrm{L}}^{\mathrm{i}}-c_{\mathrm{L}}^{0}\right)}{\operatorname{erfc}(\Lambda)} \exp \left(-\Lambda^{2}\right)-\sqrt{\varkappa} \frac{\left(c_{\mathrm{S}}^{\mathrm{i}}-c_{\mathrm{S}}^{0}\right)}{\operatorname{erfc}\left(\frac{\Lambda}{\sqrt{\varkappa}}\right)} \exp \left(-\frac{\Lambda^{2}}{\varkappa}\right) \\
& =\sqrt{\pi}\left(c_{\mathrm{L}}^{\mathrm{i}}-c_{\mathrm{S}}^{\mathrm{i}}\right) \Lambda,
\end{aligned}
$$

which depends only on the ratio $\varkappa=D_{\mathrm{S}} / D_{\mathrm{L}}$ and not on the absolute values of the diffusion coefficients. In units of atomic scale $((\AA)$ and $(\mathrm{ns}))$, the sharp interface is at the position $x_{\mathrm{f}}(\AA)=\alpha \sqrt{t(\mathrm{~ns})}$ with $\alpha=2 \Lambda \sqrt{D_{\mathrm{L}}\left(\mathrm{m}^{2} \mathrm{~s}^{-1}\right) \times 10^{11}}$. 


\section{Thermodynamic data for the phase-field simulations}

As mentioned in the introduction, the execution of phasefield simulations for real material systems requires the input of thermodynamic data such as the liquidus line, the interface thickness on atomic scale, the kinetic coefficient, the surface entropy density, the diffusion coefficients and the initial concentration profiles. In the following sections, we describe how these data are extracted from the molecular dynamics simulations.

\subsection{Crystal-melt interface: thickness, surface entropy density and kinetic coefficient}

The crystal-melt interface is diffuse, facetted and extends over several atom sizes. An approximate characterization of its position and width is, however, necessary to perform detailed analysis of the conditions of the growth process such as the growth direction and the growth velocity as a function of the undercooling. A well-defined interface profile is needed to perform quantitative phase field simulations of solidification in the binary two-phase $\mathrm{Ni}-\mathrm{Zr}$ system. In order to assign particles either to the solid or to the liquid phase, and hence to characterize the crystal-melt interface, we use the local order parameter $Q_{1}$ introduced by Steinhardt et al [18]. This parameter is known to well discriminate a crystalline environment from a liquid one. We start by defining the set of nearest neighbours of a particle $i$ to be the region of all particles $j$ within a given radius. This radius is chosen to be identical with the first minimum in the radial pair correlation function $g_{i j}(r)$. The vectors $\boldsymbol{r}_{i j}$ of joining neighbours are called bonds. The local structure around a particle $i$, having $N_{\mathrm{b}}(i)$ nearest neighbours can be characterized by

$$
Q_{l m}^{i}=\frac{1}{N_{b}(i)} \sum_{j=1}^{N_{b}(i)} Y_{\operatorname{lm}}(\theta(\boldsymbol{r}), \phi(\boldsymbol{r})),
$$

where $Y_{l m}(\theta(\boldsymbol{r}), \phi(\boldsymbol{r}))$ are the spherical harmonics and $\theta(\boldsymbol{r})$ and $\phi(r)$ are the polar and the azimuthal angles of vector $\boldsymbol{r}$. $Q_{l m}^{i}$ depends on the orientation of the reference frame. However, second-order rotationally invariant combinations can be constructed:

$$
Q_{l}^{i}=\left(\frac{4 \pi}{2 l+1} \sum_{m=-l}^{l}\left|Q_{l m}^{i}\right|^{2}\right)^{1 / 2}
$$

which allow invariant characterization of the degree of crystallization.

In this study, we use the $Q_{6}^{i}$ parameter because of its high sensitivity [18] in discriminating crystalline and liquid local environment. The sensitivity can clearly be seen in figure 2 , where we display the time average of the order-parameter $x$-profile $Q_{6}(x)$ for one equilibrium interface. $Q_{6}(x)$ varies from a liquid value of about 0.27 to a bcc-crystal value of about 0.47 within a distance of $12 \AA$. This interfacial thickness, provided by the order parameter $Q_{6}(x)$, is considered to

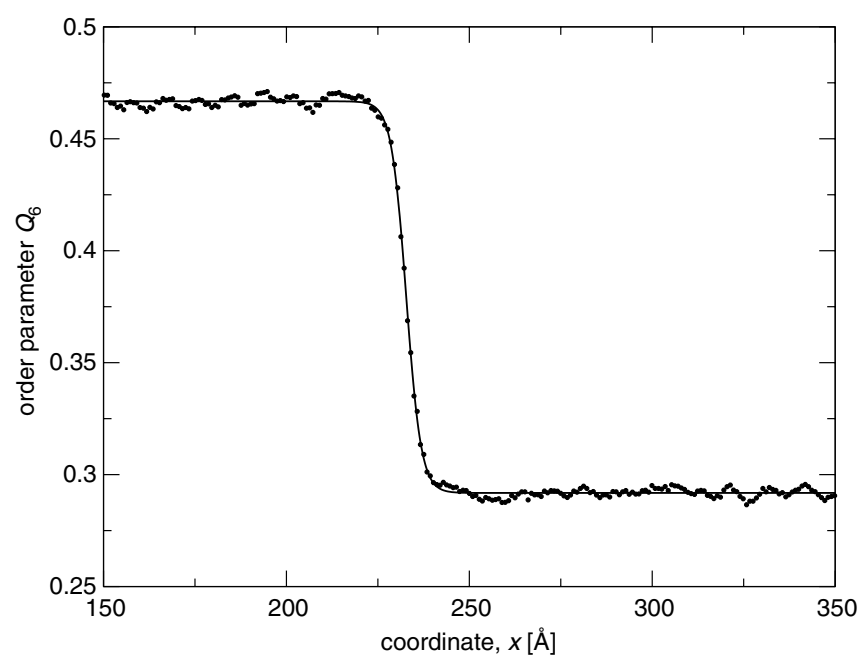

Figure 2. Fitted tanh-profile of the diffuse interface (solid line) through the data points (dots) of the order parameter $x$-profile $Q_{6}(x)$ across the solid-liquid interface at equilibrium.

approximate the interface width for the phase-field model in equation (3).

At thermodynamic equilibrium, the time derivative $\partial \varphi / \partial t$ as well as $\partial f / \partial \varphi$ in equation (3) vanish and we obtain the well-known tanh profile of the phase-field variable across the interface

$$
\psi(x)=A+\frac{B}{2}\left(1-\tanh \frac{3}{2} \frac{\left(x-x_{0}\right)}{\varepsilon}\right) .
$$

A fitting of the molecular dynamics data points yields the parameters $A=0.291808, B=0.174943, x_{0}=232.755 \AA$ and $\varepsilon=6.12755 \AA$ and the continuous profile illustrated as solid line in figure 2 . The value of parameter $\varepsilon=6.12755 \AA$ is used for the numerical solution of the phase-field equation. Herewith, the variation of the phase-field from $\varphi=0.047$ to $\varphi=0.953$ across the interface takes place within the interval of $2 \varepsilon \simeq 12 \AA$.

In the simulations, we use the value of interfacial energy $\sigma=1 \mathrm{eV} \mathrm{nm}^{-2}=0.16 \mathrm{~J} \mathrm{~m}^{-2}[19]$ and the interfacial entropy density is estimated as $\gamma=\sigma / T_{\mathrm{m}}=7.4 \times 10^{-5} \mathrm{~J} \mathrm{mK}^{-1}$. The interface mobility $v$ in the phase-field equation (equation 3 ) is connected to the kinetic coefficient $\mu$ by the relation

$$
v=\frac{\mu \sigma T_{\mathrm{m}}}{L_{\mathrm{m}}}
$$

where $L_{\mathrm{m}}$ is the latent heat. Different values of $v$ have been used in computations (see section 4).

\subsection{Bulk properties: free energy density and diffusion coefficients}

To define the free energy density $f(c, T)$ in equations (1) and (2), the function of the liquidus line $C_{\mathrm{L}}(T)$ has to be estimated. By polynomial interpolation of the molecular dynamics data in figure 3 , we get the expression

$$
C_{\mathrm{L}}(T)=-0.704\left(\frac{T}{T_{\mathrm{m}}}\right)^{2}+0.478\left(\frac{T}{T_{\mathrm{m}}}\right)+0.226,
$$

which is also included in figure 3 as a solid line. 


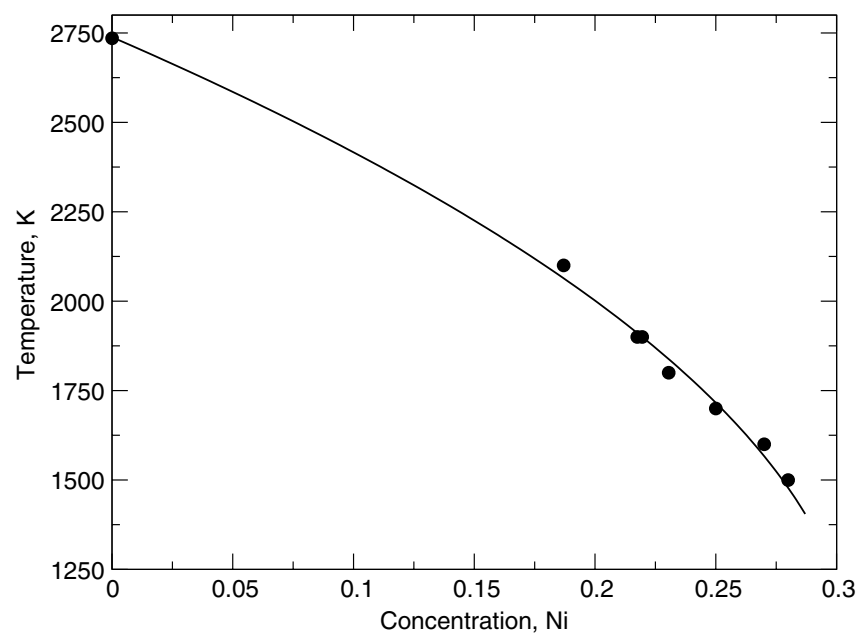

Figure 3. Sectional region of the $\mathrm{Ni}-\mathrm{Zr}$ phase diagram with discrete molecular dynamics data points (solid dots) and the interpolation function (equation (18)) used for the phase-field simulations (solid line).

In this study we are interested in the diffusion-coefficient profile across the interface along the $x$ direction. Therefore, we determine the two-dimensional diffusion coefficient $D$, that we compute using Einstein's relation between the mean-square lateral displacement of the atoms and $D$ in the asymptotic limit:

$$
\begin{aligned}
D= & \frac{1}{4 t} \lim _{t \rightarrow \infty} \partial_{t}\left\langle\left(y_{i}\left(t_{0}+t\right)-y_{i}\left(t_{0}\right)\right)^{2}+\left(z_{i}\left(t_{0}+t\right)\right.\right. \\
& \left.\left.-z_{i}\left(t_{0}\right)\right)^{2}\right\rangle_{i, t_{0}},
\end{aligned}
$$

where $y_{i}(t)$ and $z_{i}(t)$ are the lateral coordinates of particle $i$ at time $t$. The average is performed over particles $i$ and reference time $t_{0}$. The mean-square displacement (MSD) is measured until a time $t^{\star}$ when the asymptotic linear regime is reached. In the melt, $t^{\star}$ is generally comparable to the $\alpha$-relaxation time, i.e. the life time of the atom's neighbouring cages. In the temperature range considered in this work ( $T$ around $1700 \mathrm{~K}$ ), $t^{\star}$ is of a few picoseconds (compare with data of Mutiara and Teichler in [3] for $\mathrm{Ni}_{20} \mathrm{Zr}_{80}$ ). To deduce the function $D(x)$ at position $x$, we consider all particles belonging to the layer $(x-\Delta x / 2, x+\Delta x / 2)$ during the time interval $\left(t_{0}, t_{0}+t^{\star}(x)\right)$. We choose $t^{\star}=25 \mathrm{ps}$ and a layer thickness $\Delta x=1 \AA$. Since the particles can move from a layer to another during the simulation, we consider only the MSD of those atoms whose residence time in their respective layers is less than $t^{\star}$. We check the reliability of the calculated $D(x)$ by comparing its value in the bulk (far from the interface) with that of the threedimensional self-diffusion coefficient. These two quantities have to be the same due to the isotropic character of the liquid structure. This isotropy is obviously broken at the interface, but the diffusion-coefficient profile according to equation (19) is found to be compatible with the three-dimensional diffusivity averaged over the interface region defined in section 3.1. The distribution of dots in figure 4 shows the Ni-diffusion profile in the two-phase layer at $T=1700 \mathrm{~K}$ proposed by molecular dynamics simulations. A small decrease in the diffusivity is observed in the liquid ahead of the interface as a result of the confinement effect due to the crystalline wall. The noise in the crystalline region is caused by the poor statistics owing

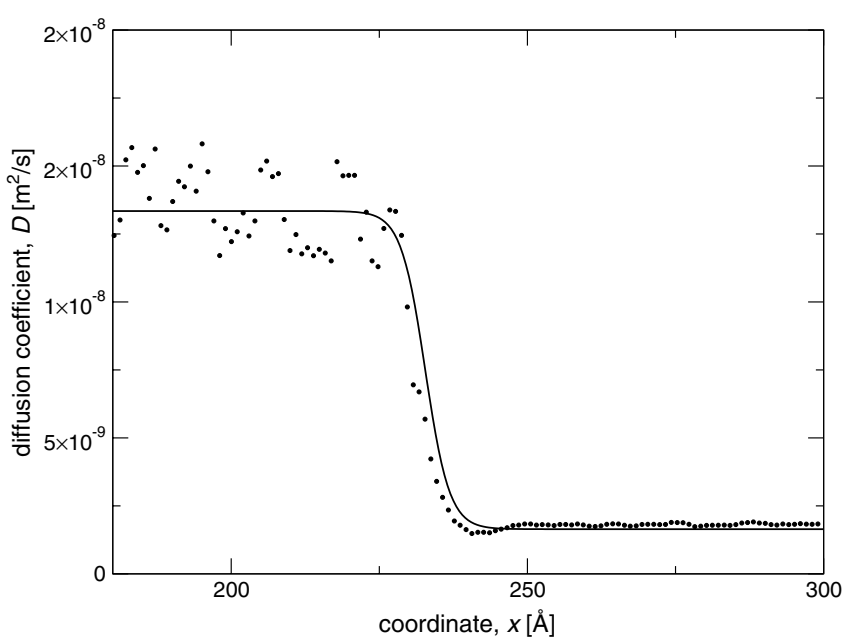

Figure 4. Diffusion of $\mathrm{Ni}$ atoms in the two phases and at the solid-liquid interface: molecular dynamics data (solid dots) and interpolated function used for the phase-field simulations (solid line).

to the very small solubility of $\mathrm{Ni}$ atoms in the $\mathrm{Zr}$ crystal $\left(C_{\mathrm{s}} \sim 0.007\right.$ at $\left.T=1900 \mathrm{~K}\right)$.

We observe a higher mobility of $\mathrm{Ni}$ atoms in the bcc $\mathrm{Zr}$ crystal than in the melt at the considered temperature. To explain this somehow striking feature, we have to compare the environment of $\mathrm{Ni}$ in both regions. In the melt, $\mathrm{Ni}$ atoms are confined in cages, which are the same as those building the intermetallic phases NiZr (B33 type with Ni-centred trigonal prisms as structural units) and $\mathrm{NiZr}_{2}$ (CrB type with Archimedean antiprisms as structural units). These structural units, dictated by the nature of the interatomic interactions when building the crystalline phases, are obviously more stable than the octahedral sites encountered by $\mathrm{Ni}$ atoms in the bcc $\mathrm{Zr}$ crystal. Such an anomalously fast diffusion of $\mathrm{Ni}$ and $\mathrm{Co}$ atoms in the bcc $\mathrm{Zr}$ crystal has been reported in earlier works (cf [17]).

In the phase-field model, the diffusion process is described by equation (4), where the diffusion of atoms in the phases is approximated by a linear interpolation function $D(\varphi)=$ $D_{\mathrm{S}} \varphi+D_{\mathrm{L}}(1-\varphi)$ of the order parameter between the values of the diffusion in the solid $D_{\mathrm{S}}$ and in the liquid $D_{\mathrm{L}}$. By fitting the molecular dynamics data, we get $D_{\mathrm{L}}=1.64368 \times 10^{-9} \mathrm{~m}^{2} \mathrm{~s}^{-1}$ and $D_{\mathrm{S}}=1.33442 \times 10^{-8} \mathrm{~m}^{2} \mathrm{~s}^{-1}$ and the profile shown as solid line in figure 4.

\subsection{Initial concentration profile}

The initial concentration field for the phase-field simulations is derived from the distribution of $\mathrm{Ni}$ atoms at $T=1900 \mathrm{~K}$ provided by the molecular dynamics computations. To evaluate the discrete molecular dynamics data and to determine the initial concentrations $c_{\mathrm{S}}^{0}$ and $c_{\mathrm{L}}^{0}$ of $\mathrm{Ni}$ in the solid and liquid phase, we consider both halves of the periodic molecular dynamics sample setting. The values $c_{\mathrm{S}}^{0}$ and $c_{\mathrm{L}}^{0}$ as well as the equilibrium partition coefficient $k_{\mathrm{e}}=c_{\mathrm{S}}^{0} / c_{\mathrm{L}}^{0}$ are given by the mean values of the concentrations on the left and right sides of the sample. The atom distributions on both halves of the 
Table 1. Parameters of the fitted interpolation function $\psi(x)$ (equation (20)) for both sides of the sample.

\begin{tabular}{llllll}
\hline Side of the sample & $\varepsilon$ & $x_{0}$ & $c_{\mathrm{S}}^{0}$ & $c_{\mathrm{L}}^{0}$ & $k_{\mathrm{e}}$ \\
\hline Left & $6.47519 \AA$ & $219.097 \AA$ & 0.00738589 & 0.21920189 & 0.03369 \\
Right & $5.75676 \AA$ & $1558.75 \AA$ & 0.00821407 & 0.21934307 & 0.0374485 \\
\hline
\end{tabular}

sample are fitted separately by a continuous profile function

$$
\psi(x)=c_{\mathrm{S}}+\frac{c_{\mathrm{L}}-c_{\mathrm{S}}}{2}\left(1+\tanh \frac{3}{2} \frac{\left(x-x_{0}\right)}{\varepsilon}\right),
$$

for which the approximated parameters $\varepsilon, x_{0}, c_{\mathrm{S}}$ and $c_{\mathrm{L}}$ are summarized in table 1.

From the data in table 1 we calculate the initialization data for the phase-field simulations by taking the mean values

$$
c_{\mathrm{S}}^{0}=0.0078, \quad c_{\mathrm{L}}^{0}=0.21927 \quad \text { and } \quad k_{\mathrm{e}}=0.0356 .
$$

\section{Simulation results}

Using the data from the molecular dynamics simulations, we compare results of the concentration profiles and of the solidification front dynamics obtained from phase-field computations, from sharp interface modelling and from molecular dynamics simulations.

The sharp interface solution in equations (10)-(13) depends on the equilibrium concentrations at the interface, on the far-field (initial) concentration and on the diffusion coefficients. These parameters are computed from the molecular dynamics data as discussed in the previous section. Utilizing these values in equation (13), we get the parameter $\Lambda=0.0758$ describing the dynamics of the front position in time. As a result, the growth velocity of the interface is clearly slower than the evolution extracted from the molecular dynamics simulations. Using ( $\AA$ ) for the length and (ns) for time scale, the molecular dynamics data on 'front position versus time' can be fitted by $x_{\mathrm{f}}(\AA)=\alpha \sqrt{t(\mathrm{~ns})}$ with $\alpha=$ $3.86 \AA \mathrm{ns}^{-1 / 2}$. For the sharp interface solution, the obtained value of $\Lambda=0.0758$ together with the diffusion coefficient $D_{\mathrm{L}}=1.64 \times 10^{-9} \mathrm{~m}^{2} \mathrm{~s}^{-1}$ leads to a smaller value of $\alpha$, namely $\alpha=1.94 \AA \mathrm{ns}^{-1 / 2}$. Based on these observations, we determine a new set of parameters by fitting the 'front position versus time' data from the sharp interface solution to the molecular dynamics data points. For that, we fix the parameter $\varkappa=8.11$ using the relationship between diffusion coefficients. From equation (13), the value of the parameter $\Lambda$ follows to be $\Lambda=0.0758$. Using this value of $\Lambda$, a new value of the diffusion coefficient in the liquid is obtained: $\tilde{D}_{\mathrm{L}}=$ $6.47 \times 10^{-9} \mathrm{~m}^{2} \mathrm{~s}^{-1}$. Equivalently, the corresponding diffusion coefficient in solid reads $\tilde{D}_{\mathrm{S}}=\varkappa \tilde{D}_{\mathrm{L}}=5.25 \times 10^{-8} \mathrm{~m}^{2} \mathrm{~s}^{-1}$. The new values of diffusion coefficients allow to match the sharp interface solution to the molecular dynamics results under the constraint of maintaining the ratio $\varkappa$ between the diffusion coefficients in solid and liquid phase.

In contrast to the sharp interface model, the phase-field model includes finite kinetics of the interface described by the interface mobility $v$. The parameter $\varepsilon$ that determines the

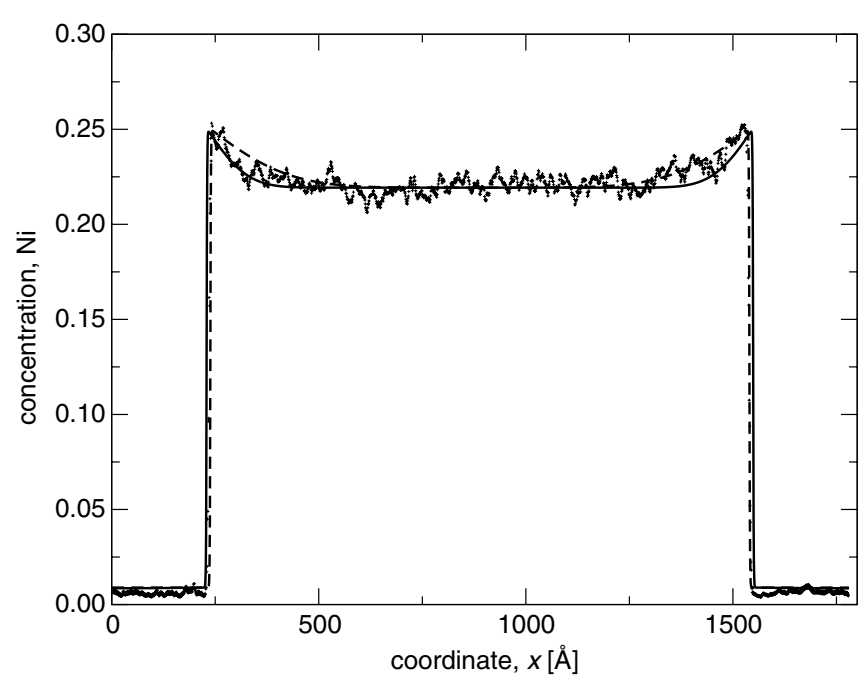

Figure 5. Concentration field of $\mathrm{Ni}$ in the solid and liquid phase of the binary $\mathrm{Ni}-\mathrm{Zr}$ system: molecular dynamics data ('+'), phase-field model with $D_{\mathrm{L}}=1.64 \times 10^{-9} \mathrm{~m}^{2} \mathrm{~s}^{-1}$ (solid line) and with $D_{\mathrm{L}}=6.47 \times 10^{-9} \mathrm{~m}^{2} \mathrm{~s}^{-1}$ (dashed line)

thickness of the diffuse interface follows from the fitting to the molecular dynamics order parameter. The interface entropy density $\gamma$ plays a role mainly for curved interfaces and in the considered application and sample setup, we focus on planar interfaces.

Based on the approximation of the phase-field parameters by molecular dynamics data, the concentration profiles and the interface dynamics are compared. In figure 5, the symbol ' + ' shows the molecular dynamics concentration profile of $\mathrm{Ni}$ atoms after $20 \mathrm{~ns}$ of the solidification process. The solid line represents the phase-field solution employing the diffusion coefficients $D_{\mathrm{L}}=1.64 \times 10^{-9} \mathrm{~m}^{2} \mathrm{~s}^{-1}$ and $D_{\mathrm{S}}=$ $1.33 \times 10^{-8} \mathrm{~m}^{2} \mathrm{~s}^{-1}$ proposed by the molecular dynamics computations. Figure 6 shows the region around the left solid-liquid interface after $20 \mathrm{~ns}$ of the solidification process. For these values of the diffusion coefficients, we find in analogy with the sharp interface solution that the phasefield simulations of the front position in time performs a mismatch with the molecular dynamics behaviour. However, the concentration profile shows a reasonable agreement with molecular dynamics distribution of $\mathrm{Ni}$ atoms. The analysis of this effect has shown that an increase in the interface mobility (and correspondingly kinetic coefficient) by three orders of magnitude from $v=2.5 \times 10^{-10} \mathrm{~m}^{2} \mathrm{~s}^{-1}$ to $v=$ $10^{-7} \mathrm{~m}^{2} \mathrm{~s}^{-1}$ changes the dynamic curve, but does not establish an agreement with the molecular dynamics data. Figure 7 displays the molecular dynamics data (' + ') and the phase-field model results for the two values of the interface mobility: $v=$ $2.5 \times 10^{-10} \mathrm{~m}^{2} \mathrm{~s}^{-1}$ (dashed line) and $v=10^{-7} \mathrm{~m}^{2} \mathrm{~s}^{-1}$ (dashdotted line). The further increasing of the interface mobility 


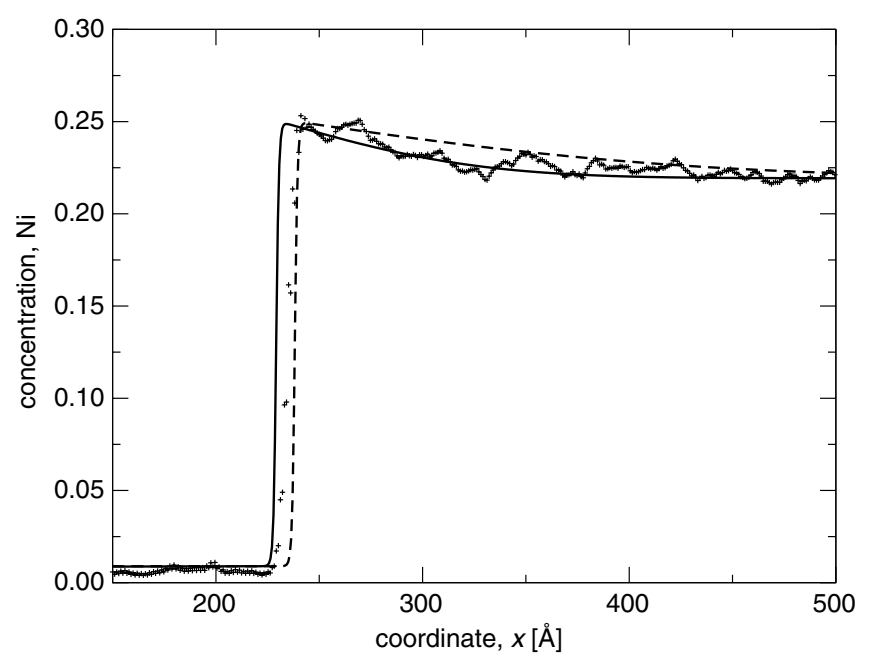

Figure 6. Concentration field of $\mathrm{Ni}$ around the left solid-liquid interface: molecular dynamics data ('+'), phase-field model with $D_{\mathrm{L}}=1.64 \times 10^{-9} \mathrm{~m}^{2} \mathrm{~s}^{-1}$ (solid line) and with $D_{\mathrm{L}}=6.47 \times 10^{-9} \mathrm{~m}^{2} \mathrm{~s}^{-1}$ (dashed line).

above $v=10^{-7} \mathrm{~m}^{2} \mathrm{~s}^{-1}$ leads only to insignificant changes of the interface dynamics. Following the suggestion of the sharp interface predictions, we applied the modified diffusion coefficients $\tilde{D}_{\mathrm{L}}$ and $\tilde{D}_{\mathrm{S}}$ together with $v=10^{-7} \mathrm{~m}^{2} \mathrm{~s}^{-1}$ as initialization parameters for the phase-field simulation. For this data set, we obtain an excellent agreement between the molecular dynamics and the phase-field results (solid line in figure 7) and conclude that a four times larger diffusion coefficient is necessary to match the characteristic dynamics of both methods. The concentration profiles associated with the modified diffusion coefficients also provide a good agreement with the molecular dynamics data curve as illustrated by dashed lines in figures 5 and 6 . The root-mean-square deviation between the molecular dynamics data and the phase-field profile in the region near the left solid-liquid interface has the value 0.022 for the diffusion coefficient $D_{\mathrm{L}}=1.64 \times$ $10^{-9} \mathrm{~m}^{2} \mathrm{~s}^{-1}$ and the value 0.016 for the modified diffusion coefficient $D_{\mathrm{L}}=6.47 \times 10^{-9} \mathrm{~m}^{2} \mathrm{~s}^{-1}$.

\section{Summary and concluding remarks}

The present contribution is concerned with the propagation of the planar solidification front in a two-phase crystalliquid structure under non-equilibrium conditions. It compares results from the molecular dynamics simulations with those of phase-field modelling.

Such a comparison is optimally suited to estimate from molecular dynamics simulations the phenomenological and crucial parameter $v$ of interface mobility. In the phase-field model, there are further parameters that have to be imported from outside, such as the interface thickness $\varepsilon$, the diffusion coefficients $D_{\mathrm{S}}$ and $D_{\mathrm{L}}$ of the solute in solid and liquid phase, and the equilibrium partition coefficient $k_{\mathrm{e}}$. As demonstrated, these parameters can be extracted from molecular dynamics simulations of a finite-temperature crystal-liquid sandwich with an interface under equilibrium conditions, while $v$ needs information about non-equilibrium interface dynamics.

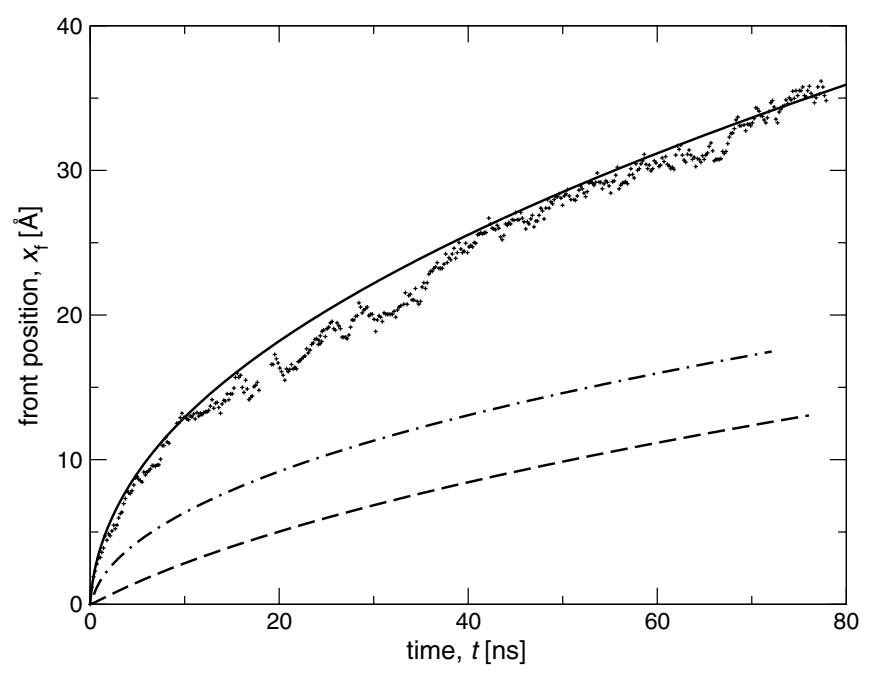

Figure 7. Comparison of the computed interface position as a function of time obtained from a molecular dynamics simulation (discrete data points) and from phase-field computations with different data sets. The dashed line corresponds to input data: $D_{\mathrm{L}}=1.64 \times 10^{-9} \mathrm{~m}^{2} \mathrm{~s}^{-1}, D_{\mathrm{S}}=1.33 \times 10^{-8} \mathrm{~m}^{2} \mathrm{~s}^{-1}$ and $v=2.5 \times 10^{-10} \mathrm{~m}^{2} \mathrm{~s}^{-1}$, the dotted-dashed line shows the result for a larger kinetic coefficient of $v=10^{-7} \mathrm{~m}^{2} \mathrm{~s}^{-1}$ and the solid line represents the simulated curve for $\tilde{D}_{\mathrm{L}}=6.47 \times 10^{-9} \mathrm{~m}^{2} \mathrm{~s}^{-1}$, $\tilde{D}_{\mathrm{S}}=5.25 \times 10^{-8} \mathrm{~m}^{2} \mathrm{~s}^{-1}$ and $v=10^{-7} \mathrm{~m}^{2} \mathrm{~s}^{-1}$.

Regarding the concentration profile and propagation velocity of the solidification front in the non-equilibrium situation, our analysis yields that one needs increasing the effective diffusion coefficients in the phase-field model by a factor of four against their molecular dynamics estimates in order to get acceptable agreement between the applied phase-field model and the molecular dynamics simulation. This finding makes obvious that bridging the gap between molecular dynamics simulations and phase-field model treatment cannot be achieved by simply exchanging parameters, but one has to consider there effects in the context of the inevitably different assumptions and approximations of the methods. At present, one can only speculate about the reason for the discrepancy in the diffusion coefficients. Regarding this, there remains, for example, the question for the role of activity coefficients in the chemical potential of the liquid and crystal phases. An answer to this question is, however, clearly outside the range of our present analysis, as it needs information, e.g. about the detailed concentration dependence of the free enthalpy of the liquid phase.

\section{Acknowledgments}

This work was supported by the German Research Foundation (DFG) under the priority research program 1120: 'Phase transformations in multicomponent melts', Grant No. Ne 822/3-3 and $\mathrm{Te}$ 42/7-3. The funding is gratefully acknowledged.

The computations have been carried out at the high performance computer centre Hannover (HLRN). 


\section{References}

[1] Boettinger W J, Warren J A, Beckermann C and Karma A 2002 Annu. Rev. Mater. Res. 32163

[2] Küchemann K-B 2004 Dr rer. nat. Thesis University of Göttingen

[3] Teichler H 1996 Phys. Rev. Lett. 7662

Mutiara A B and Teichler H 2001 Phys. Rev. E 64046133

Ladawa I and Teichler H 2006 Phys. Rev. E 73031501

[4] Massalski T B (ed) 1990 Binary Alloy Phase Diagrams (Metals Park, OH: ASM International)

[5] Chen L-Q 2002 Annu. Rev. Mater. Res. 32113

[6] Bi Z and Sekerka R F 1998 Physica A 26195

[7] Garcke H, Nestler B and Stinner B 2004 SIAM J. Appl. Math. 64775

[8] Bragard J, Karma A, Lee Y H and Plapp M 2002 Interface Sci. 10121
[9] Hoyt J J, Sadigh B, Asta M and Foiles S M 1999 Acta Mater. 473181

[10] Wu K-A and Karma A 2007 Phys. Rev. B 76184107

[11] Stillinger F H and Weber T A 1985 Phys. Rev. B 315262

[12] Hausleitner Ch and Hafner J 1992 Phys. Rev. B 45115

[13] Teichler H 1999 Phys. Rev. B 598473 (References on $\mathrm{p} 6$ and $\mathrm{p} 7$ )

[14] Voigtmann Th, Meyer A, Holland-Moritz D, Stüber S, Hansen T and Unruh T 2008 Europhys. Lett. 8266001

[15] Teichler H 1997 Defect Diffus. Forum 143-147 717

[16] Teichler H 2001 J. Non-Cryst. Solids 293339

[17] Averback R S 1991 Mater. Res. Bull. 1647

[18] Steinhardt P J, Nelson D R and Ronchetti M 1983 Phys. Rev. B 28784

[19] Vinet B et al 2002 J. Coll. Surf. Sci. 255363 